\title{
A NEW FORMULATION OF CANONICAL PERTURBATION THEORY
}

DAVID P. STERN

MAY 1970
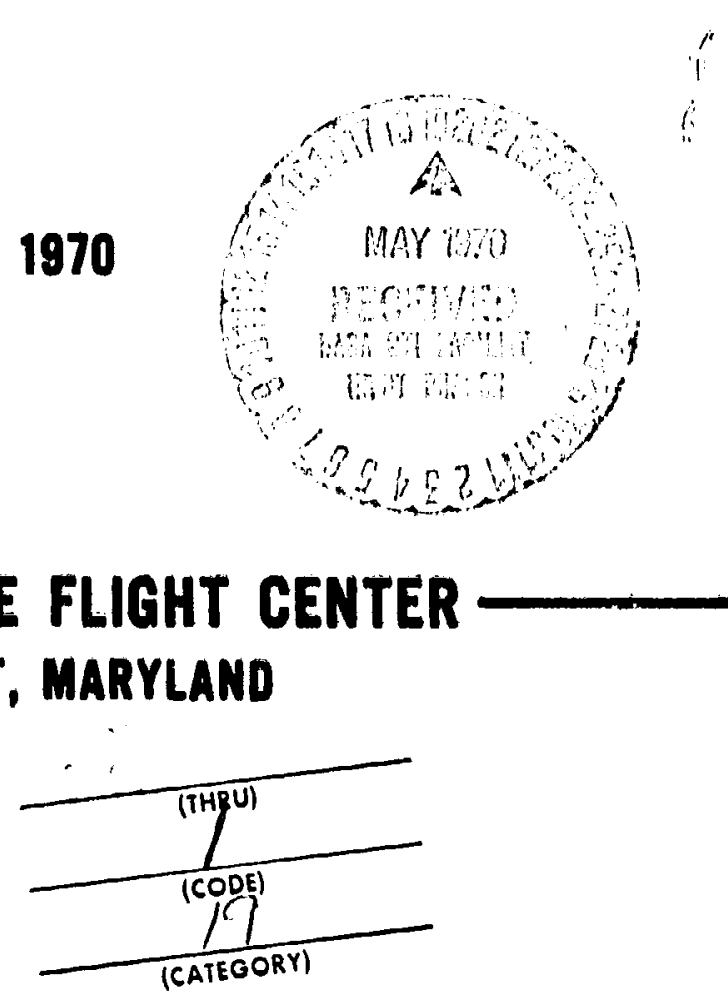
A New Formulation of Canontcal P'srturbation Theory

David P. Stern

Laboratory for Space Physics

Goddard Space Flight Center

Groenbelt, Maryland 20771

Abstrect

By means of direct canonical transformations, the Poincare-

Von Zeipel perturbation mothod may be modifled into a more useful form.

Two particular variants of this approach are examined and explicit

formulas are given for carrying them out to at least the 4-th order. 


\section{INTRODUCTION}

In this work, a modification of the hamilton-Jacobi equation is developed, utilizing the direct formulation of canonical transformations. The resul ting perturbation schome is easy to apply and may bo related oither to the standard Poincaró-Von Zeipel method or to Hord's expansion, depending on the choice of certain expressions entering it.

The solution of a mechantcal problem by the Hambiton-Jacobi equation seeks a canontcal transformation to now variables, in wich tize complezity of the problem is reduced. In partioular, let us consider a perturbed periodic motion, with $p_{1}$ the action ramiable of the unperturbed motion and with a time-independent Hamiltonlan

$$
\text { 目 }=p_{1}+\sum_{k=1} \varepsilon^{k}{ }_{R}^{(k)}(\underline{p}, \underline{q})
$$

Then by the Poincare-Von Zeipel method (Poincere, 1893; Von Zeipel, 1916; Corben and Stehle, 1960) one seeks a near-identity transformation to new variables $(\underline{p}, Q)$, given by the generating function

$$
\sigma=\sum p_{i} q_{1}+\sum_{k=1} \varepsilon^{k} \sigma^{(k)}(\underline{p}, \underline{q})
$$

via

$$
\left.\begin{array}{l}
\partial \sigma / \partial p_{1}=a_{1} \\
\partial \sigma / \partial q_{1}=p_{1}
\end{array}\right\}
$$

In this method, one uses the fact that the ner Hamiltonian equals the old one, substituting (3) to obtain

$$
B(\partial \sigma / \partial \underline{q}, \underline{q})=\mathbb{B}^{*}(\underline{\mathcal{P}}, \partial \sigma / \partial \underline{\underline{P}})
$$


Whon this is expanded in orders of $\varepsilon$, a recurative scheme is obtained and both $\mathrm{B}^{*}$ and $\sigma$ may be derived order by oxder, subject to the condition that the transformod angle variable $Q_{1}$ is absent from the new Harilitonian $H^{*}$. When this is accompliahed, not only has $Q_{1}$ been romoved from the problen, but its conjugate $P_{1}$ has become a constant of the motion, so that the motion represented by $H^{*}$ has two fewor variables to contend with.

Now a near-1dentity transformation may be represented in other ways than by oquations (2)-(3); these ways may offer more convenience, since $\sigma$ in (2) depende on both old and new variables and therefore the relations (3) require further untangling to be useful. In perticular, we may consider a direct canonical transformation

$$
\underline{z}=\underline{y}+\sum_{k=1} \varepsilon^{k} \underline{\zeta}^{(k)}(\underline{y})
$$

where we will use the notation

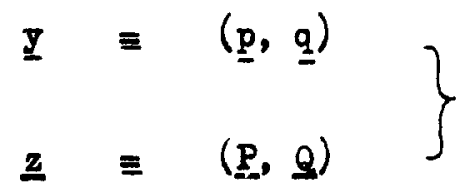

with

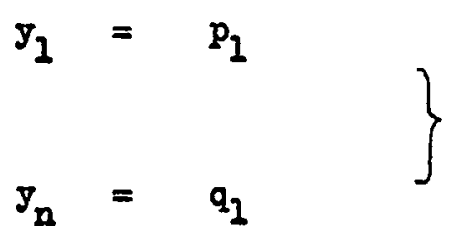

Also, it wall be useful to denote by a tilde the vector formed by omitting the component $y_{n}$ or the one corresponding to it, e.g.

$$
\underline{z} \equiv\left(\tilde{E}, z_{n}\right)
$$

Then, if one substitutes (5) into the basic relation 


$$
H(\underline{y})=H^{*}(\underline{\underline{z}})
$$

and expands order by order, the appropriate transformation (5) may be derived. Hord (1966) used this approach, with (5) given through Iste's formulation of canonical transfurmations (Le, 1838). Here a oomewhat different approach (Storm, 1970) will be used, wich can be made equivalent either to Horl'B or to the PoincareVon Zeipel method, depending on one's choice of the expreseion $\underline{f}^{(k)}$, defined later.

\section{NOTATION}

If the position vector $I$ in phase space is defined as in (6), let its conjugate $\overline{\bar{y}}$ be defined

$$
\bar{y}=(\underline{q},-\underline{p})
$$

from wich, by (7)

$$
\left.\begin{array}{l}
\bar{y}_{1}=y_{n} \\
\bar{y}_{n}=-y_{1}
\end{array}\right\}
$$

Also, if $\nabla$ operates in $y$-space, we can define there a conjugate gradient operator $\bar{\nabla}$, the ith component of which is $\partial / \partial \bar{y}_{1}$.

To express functions of $\underline{z}$ in terms of $z$, we shall use Taylor expansion operators $\mathrm{T}^{(\mathrm{k})}$ (Musen, 1965), with operation denoted (for clarity) by an asterisk:

$$
\begin{aligned}
G(\underline{z}) & =G\left(y+\sum \varepsilon^{k} \underline{\zeta}^{(k)}\right) \\
& =\exp \left(\sum_{k=1} \varepsilon^{k} \underline{\zeta}^{(k)} \cdot \nabla\right) * G(\underline{y}) \\
& =\sum_{k=0} \varepsilon^{k} T^{(k)} * G(\underline{y})
\end{aligned}
$$

The $T^{(k)}$ may be obtained by expanding the exponential and regrouping terms according to their order in $E$; the first few of them are 


$$
\begin{aligned}
& T^{(0)}=1 \\
& T^{(1)}=\underline{\zeta}^{(1)} \cdot \nabla \\
& T^{(2)}=\underline{\zeta}^{(2)} \cdot \nabla+\frac{1}{2} \underline{\zeta}^{(1)} \underline{\zeta}^{(1)}: \nabla \nabla \\
& T^{(3)}=\underline{\zeta}^{(3)} \cdot \nabla+\underline{\zeta}^{(1)} \underline{\zeta}^{(2)}: \nabla \nabla+(1 / 6) \underline{\zeta}^{(1)} \underline{\zeta}^{(1)} \underline{\zeta}^{(1)}: \nabla \nabla \nabla
\end{aligned}
$$

and so forth. In general one can mite

$$
T^{(k)}=\underline{\zeta}^{(k)} \cdot \nabla+N^{(k)}
$$

where $\mathrm{N}^{(\mathrm{k})}$ is an operator involving $\nabla$ at least twice.

\section{THE TRANSPOBMATION}

The relation (5) holds for any near-identity transformation. If $\Lambda^{\text {is }}$ to be a canonical transformation, $\underline{\zeta}^{(k)}$ most satiofy adidtional requirements, and it may be shown (Stern, 1970) that these always have the general form

$$
\underline{\zeta}^{(k)}=\underline{\underline{\underline{e}}}^{(k)}(\underline{\zeta})+\bar{\nabla} x^{(k)}
$$

where $\underline{\underline{\underline{f}}}^{(k)}(\underline{\zeta})$ are expressions involving lower orders and $\chi^{(k)}$ is the $k$-th oxder of a generalization of the generating function. Kany alterative choices of $f^{(k)}$ are possible (one can often construct lower oxder expressions that have the form of a conjugate gradient and add them to $\underline{\underline{f}}^{(k)}$ ) but wo shall be concerned with two ia particular. One may choose

$$
f_{1}^{(k)}=-\sum_{m=1}^{k-1} s^{(m)} *\left(\partial x^{\left.(k-n) / \partial \bar{y}_{1}\right)}\right.
$$


where $s^{(m)}$ are operators resembling the $\mathrm{m}^{(m)}$ of (13) but with $\zeta^{(j)}$ everywhere replaned by

$$
\pi^{(j)}=\left(\zeta_{1}^{(j)}, \ldots \zeta_{(n / 2)}^{(j)}, 0, \ldots 0\right)
$$

that 1.8, with rectors in wich the momentum-1ike parts are retained and the coordinate-2ike parta are roplaced by zeros. It may then be shom (see Sterm, 1970, where this choice of ${\underline{f^{(k)}}}^{(k) \text { denoted by }} \underline{\rho}^{(k)}$ ) that in this case

$$
\chi^{(k)}=\sigma^{(k)}
$$

with $\sigma^{(k)}$ the $k$-th oxier "conventional" generating function of equation (2) which describes the same transformation. Since $s^{(m)}$ contains orders of $\zeta$ and of $\chi$ lower than the $k$-th, equation (16) must be derived recursively order by order. Alternatively, $f_{i}^{(k)}$ may be derived without breaking up $\underline{\zeta}^{(k)}$ into momentum-like and coordinate-like parts. The general method for doine this (Stern, 1970) is too lonethy to be described here, and we shall merely give the lowest orders of the result:

$$
\begin{aligned}
& \underline{\underline{f}}^{(1)}=0 \\
& \underline{\underline{\underline{I}}}^{(2)}=\frac{1}{2}^{(1)} \cdot \nabla \underline{\zeta}^{(1)} \\
& \underline{\underline{f}}^{(3)}=\underline{\zeta}^{(2)} \cdot \nabla \underline{\zeta}^{(1)} \\
& \underline{\underline{\underline{q}}}^{(4)}=\underline{\zeta}^{(3)} \cdot \nabla \underline{\zeta}^{(2)}+\frac{1}{2} \underline{\zeta}^{(2)} \cdot \nabla \zeta \underline{\zeta}^{(2)}+ \\
& +t\left[\left(\underline{\zeta}^{(1)} \cdot \nabla \underline{\zeta}^{(1)}\right) \cdot \nabla \underline{\zeta}^{(2)}-\underline{\zeta}^{(2)} \cdot \nabla\left(\underline{\zeta}^{(1)} \cdot \nabla \underline{\zeta}^{(1)}\right)\right] \\
& -t\left[\left(\underline{\zeta}^{(1)} \cdot \nabla \underline{\zeta}^{(1)}\right) \cdot \nabla \underline{\zeta}^{(1)}\right] \cdot \nabla \underline{\zeta}^{(1)}
\end{aligned}
$$


With this cholce it may be shom (Stern, 1970) that

$$
\chi^{(k)}=-s^{(k)}
$$

where $s^{(k)}$ 18 the $k$-th order generating function defined by Horl (1966).

\section{THE PERIURRATION METHOD}

Expanding (9), and using (1) and (12), g1ves

$$
\begin{aligned}
y_{1}+\sum_{k=1} \varepsilon^{k} H^{(k)}(\underline{y}) & =\sum_{k=0} \varepsilon^{k} H^{*(k)}(\underline{\tilde{z}}) \\
& =\sum_{k=0} \varepsilon^{k} H^{*(k)}\left(\underline{\tilde{y}}+\sum \varepsilon^{k} \underline{\tilde{\zeta}}^{(k)}\right) \\
& =\sum_{k=0} \varepsilon^{k} \sum_{m=0}^{k} T_{* H^{*}(k-m)}^{(\tilde{y})}
\end{aligned}
$$

This may be equated order by order, the zeroth order being simply

$$
\mathrm{H}^{*(0)}(\underline{\tilde{y}})=\mathrm{H}^{(0)}(\underline{y})=\mathbf{y}_{1}
$$

In the $O\left(\varepsilon^{k}\right)$ relation we separate the terms with II equal to 0 and $k$ from the rest and use (14) and (22). This gives

$$
\begin{aligned}
\mathrm{H}^{(k)}(\underline{y})= & \mathrm{H}^{*(k)}(\underline{\tilde{y}})+\underline{\zeta}^{(k)} \cdot \nabla y_{1}+\mathrm{I}^{(k)} * \mathrm{y}_{1}+ \\
& +\sum_{m=1}^{k-1} \mathrm{~m}^{(\mathrm{m})} * \mathrm{H}^{*(\mathrm{k}-\mathrm{m})}(\underline{\tilde{y}})
\end{aligned}
$$

In the last equation, $\mathrm{N}^{(\mathrm{k})}$ may be dropped, since it contains $\nabla$ twice or wore and its action on $y_{1}$, thus ylelde zero. Also, by (II) 


$$
\zeta^{(k)} \cdot \nabla y_{2}=\zeta_{1}^{(k)}=f_{1}^{(k)}+\partial x^{(k)} / \partial y_{n}
$$

The fina? reault, which is the basic relation of our perturbation scheme, may thus be written

$$
\partial x^{(k)} / \partial J_{n}+H^{*(k)}(\underline{\tilde{y}})=\Lambda^{(k)}(\underline{y})
$$

where

$$
\Lambda^{(k)}(\underline{y})=H^{(k)}(\underline{y})-f_{1}^{(k)}(\underline{\underline{S}})-\sum_{m=1}^{k-1} \mathrm{~T}^{(m)} \cdot \mathrm{H}^{*(k-\mathrm{k})}(\underline{\tilde{y}})
$$

If the variable $y_{n}$ enters only as an angle aith basic period unity, $\partial \chi^{(k)} / \partial y_{n}$ is clearly periodic, since any nonperiodic part of $\chi^{(k)}$ is removed by the differentiation. If one defines an averaging operator

$$
\left\langle\Lambda^{(k)}\right\rangle=\int_{0}^{1} \Lambda^{(k)} d y_{n}
$$

then clearly

$$
\begin{gathered}
\mathrm{H}^{*(\mathrm{k})}\left(\underline{\tilde{y})}=\left\langle\Lambda^{(k)}\right\rangle\right. \\
\chi^{(k)}=\int_{0}^{y_{n}}\left(\Lambda^{(k)}-\left\langle\Lambda^{(k)}\right\rangle\right) d y_{n}^{\prime}+\mu^{(k)}(\underline{\tilde{y}})
\end{gathered}
$$

where $M^{(k)}(\tilde{y})$ is an arbitrary function, representing the fact that when $y_{1}$ and $y_{12}$ are eliminated, the other variables may also undergo an arbitrary near-identsty traneformation among themselves. If no other considerations excist, it may be set equal to mero. 
Suppose the caloulation has been carried out up to and including order $(k-1)$. We now form $\Lambda^{(k)}$ by $(26)$, derive $H^{*(k)}$ and $\chi^{(k)}$ by (28) and (29), and then obtain $\zeta^{(k)}$ from $(15)$, usilkg the appropriate choice of $\underline{1}^{(k)}$. The derivation is now complete to the $\mathrm{k}$-th oxder.

\section{ACKNYKLLDGEMEANT}

The author wishes to thank Dr. Peter Musen for a stimulating discussion of this work.

REFTRRDICES

Corben, H.C., and Stehle, P.: 1960, Classical Mechanjics, 2nd Ed., John Wiley and Sons; section 75 .

Horf, G.-I.: 2966, Theory of General Perturbations with Unspecified Canonical Variables, Publ. Astron。Soc. Japan 18, 287-296

Lie, S.: 1888, Theorie der Transformationgruppen, Teubner, Leipzig

Musen, P.: 1965, On the High Order Bffects in the Methods of Krylov-Bogoliubov and Poincaré, J. Astronautical Sciences 12, 129-134

Poincaré, H.,: 1893, Les Methodes Nouvelles de la Mecanique Celeste, vol. II, Gauthler-Villars, Parle; reprinted by Dover Bublications, New York, 1957; NASA translation TI P-451, 1967.

Stern, D.: 1970, Direct Canonical Transformations, to appear in Jo siath. Physics Von Zeipel, H.: 1916, Ark. Astr. Mat. Fys. 11, no. 2 